\title{
"I'm a Survivor": Perceptions of Chronic Disease and Survivorship Among Individuals in Long-Term Remission from Opioid Use Disorder
}

\author{
Jarratt D. Pytell, MD, MHS ${ }^{1,2}{ }^{\oplus}$, Michael D. Sklar, $M A^{3}$, Joseph Carrese, MD, MPH', , \\ Darius A. Rastegar, MD2, Christine Gunn, $P h D^{5}$, and Geetanjali Chander, MD, MPH ${ }^{1,6}$
}

\begin{abstract}
'Department of Medicine, Division of General Internal Medicine, Johns Hopkins University School of Medicine, Baltimore, MD, USA; ${ }^{2}$ Department of Medicine, Division of Addiction Medicine, Johns Hopkins Bayview Medical Center, Baltimore, MD, USA; ${ }^{3}$ Department of Psychiatry and Behavioral Sciences, Johns Hopkins Bayview Medical Center, Baltimore, MD, USA; ${ }^{4}$ Berman Institute of Bioethics, Johns Hopkins University, Baltimore, MD, USA; ${ }^{5}$ Department of Medicine, Section of General Internal Medicine, Boston University School of Medicine, Boston, MA, USA; ${ }^{6}$ Department of Epidemiology, Johns Hopkins Bloomberg School of Public Health, Baltimore, MD, USA.
\end{abstract}

BACKGROUND: While opioid use disorder (OUD) is prevalent, little is known about what patients with OUD in sustained remission think about the chronic disease model of OUD and their perspectives of the cause, course, and ongoing treatment needs of their OUD.

OBJECTIVE: To (1) examine patient perceptions of the chronic disease model of addiction and disease identity and (2) use an explanatory model framework to explore how these perceptions inform ongoing treatment needs and help maintain abstinence.

DESIGN: Qualitative study of a cross-sectional cohort of patients with OUD in long-term sustained remission currently receiving methadone or buprenorphine. Participants completed a single in-depth, semi-structured individual interview.

PARTICIPANTS: Twenty adults were recruited from two opioid treatment programs and two office-based opioid treatment programs in Baltimore, MD. Half of the participants were Black, had a median (IQR) age of 46.5 (43-52) years and the median (IQR) time since the last nonprescribed opioid was 12 (8-15) years.

APPROACH: Hybrid deductive-inductive thematic analysis of the transcribed interviews.

KEY RESULTS: Some participants described a chronic OUD disease identity where they continue to live with OUD. Participants who maintain an OUD identity describe inherent traits or predetermination of developing OUD. Maintaining a disease identity helps them remain vigilant against returning to drug use. Others described a post-OUD/survivor identity where they no longer felt they had OUD, but the experience remains. Each perspective informed attitudes about continued treatment with methadone or buprenorphine and strategies to remain in remission.

CONCLUSIONS: The identity that people with OUD in sustained remission maintain was the lens through which they viewed other aspects of their OUD including cause and ongoing treatment needs. An alternative, post-

Prior Presentations This work was presented at the Society of General Internal Medicine 2020 Annual Meeting and the College on Problems of Drug Dependence 2020 Annual Scientific Meeting.

Received January 5, 2021

Accepted May 7, 2021

Published online May 23, 2021
OUD/survivorship model emerged or was accepted by participants who did not identify as currently having OUD. Understanding patient perspectives of OUD identity might improve patient-centered care and improve outcomes.

KEY WORDS: opioid use disorder; buprenorphine; methadone; chronic disease; survivorship.

J Gen Intern Med 37(3):593-600

DOI: $10.1007 / \mathrm{s} 11606-021-06925-\mathrm{Z}$

(๑) Society of General Internal Medicine 2021

\section{INTRODUCTION}

The impact of opioid use disorder (OUD) in the USA is farreaching, affecting over 2 million adults and with over 46,000 opioid overdose deaths annually in 2017 and 2018. ${ }^{1,2}$ Opioid agonist therapy (OAT) with buprenorphine or methadone is an evidence-based treatment for OUD. ${ }^{3}$ Formal treatment, including OAT and other recovery activities, enables individuals to meet treatment goals and achieve sustained remission, defined as $\geq 12$ months without meeting DSM-5 criteria for OUD. ${ }^{4}$ Studies in primary care settings and longitudinal cohorts suggest that between 1 and $3 \%$ of the adult population have a lifetime history of OUD. ${ }^{5,6}$ These patients regularly interact with the healthcare system as they seek care for medical or psychiatric comorbidities, routine preventative health, or to receive OAT. ${ }^{7}$

Patient-centered care is described as "care that is respectful and responsive to individual patient preferences, need, and values." A patient-centered care approach is associated with increased trust in clinicians, improved health outcomes, and reduced health care utilization. ${ }^{9,10}$ This approach can be particularly beneficial for patients with substance use disorders given high rates of trauma and stigma associated with substance use. ${ }^{11}$ Developing a therapeutic alliance, a measure of patient-centered care, is associated with improved engagement and retention in substance use disorder treatment. ${ }^{12}$ Most 
importantly, patient-centered care is generally what patients want when seeking care for OUD. ${ }^{13,14}$

Understanding patient perspectives is a fundamental component of patient-centered care. ${ }^{8,11,15,16}$ A patient's identity and how it relates to their chronic disease can inform their perspectives of the cause, anticipated course, and best treatment. This has been described as a patient's "explanatory model." ${ }^{17}$ Eliciting a patient's explanatory model of OUD is particularly relevant for primary care settings since patients with OUD in remission have a higher burden of medical comorbidities $^{18}$ which require ongoing, longitudinal care. Given the complex needs of this vulnerable population, there is a need to understand the explanatory models of patients with OUD in long-term remission and how it informs their perspectives on the chronic disease model and their history with OUD. To investigate this, we conducted a qualitative study of patients with OUD in long-term, sustained remission who are currently receiving OAT.

\section{METHODS}

Semi-structured individual interviews were conducted between August 2019 and July 2020 by one author (JDP). Recruitment and interviews occurred at four sites: two academic primary care clinics, one providing office-based opioid treatment (OBOT) with buprenorphine since 2003 and an HIV primary care clinic offering OBOT since 2008, and two opioid treatment programs (OTP), one was an academic and the other community-based, offering both buprenorphine and methadone. All sites are in Baltimore, MD. Eligibility criteria included English speaking, age between 21 and 65, diagnosed with OUD, currently receiving OAT, and with no self-reported non-prescribed opioid use for $\geq 5$ years. The lower bound of the age range represents the youngest possible age a participant could have $\geq 5$ years with no self-reported non-prescribed opioid use and recieve OAT. The upper age limit eliminated the role that initiating Medicare could have on the continued provision of OAT. Other substance use, including alcohol and tobacco, were not reasons for exclusion. We used maximum variation sampling to obtain a diverse study population in terms of age, race, gender, treatment location (OTP vs OBOT), and OAT medication.

Clinicians, including physicians (three of whom are authors; JP, DR, and GC), psychologists, and counselors, at the four sites identified patients who met eligibility criteria. Clinicians approached potential participants, described the study, and if a patient was interested, referred them to the study authors. Potential participants were screened by the study author (JP) to verify eligibility and the study and procedures were described in detail. Participants' verbal consent was obtained by a study author (JP) and interviews were audio-recorded and professionally transcribed. To protect anonymity, potentially identifiable information was removed or changed in participant quotes and participants were assigned a pseudonym followed by their age by decade, race, and selfreported gender. This study was approved by a Johns Hopkins School of Medicine Institutional Review Board.

Interviews focused on two topics. First, we devised interview questions to address elements of Kleinman's explanatory model of illness framework to elicit participants' perspectives of the cause, or origin, of their OUD and the appropriate treatment course. ${ }^{17,19,20}$ Second, we focused on the course of OUD and explored perceptions and acceptability of chronic disease model of OUD and current disease identity. In addition, we described a "survivorship" model of OUD and explored perceptions and acceptability of this alternative model. The theoretical framework for a survivorship model was based on the cancer survivorship model and the authors' clinical experiences. ${ }^{21}$ An initial interview guide was developed by the authors and was iteratively revised after feedback from general internal medicine and addiction medicine researchers, an Associate Professor of International Health as a part of a qualitative research methods course, and after each interview (Supplement 1).

Two authors (JDP and MDS) independently analyzed four initial transcripts with open coding, constructed a preliminary codebook, and then reanalyzed the transcripts using the codebook. Subsequent transcripts were independently coded in parallel by JDP and MDS with regular meetings to compare coding to ensure consistency, adjust the codebook as necessary, and discuss emerging themes. The transcripts were analyzed using a hybrid deductive and inductive approach to identify emerging themes. ATLAS.ti 8 (2019), a qualitative software package, was used to organize data and facilitate analysis.

\section{RESULTS}

A total of 37 potential participants were referred to participate. We were unable to establish contact after three attempts with 11 participants, three participants were contacted but interviews could not be scheduled due to repeated time conflicts, and three participants were not contacted after thematic saturation was reached. No contacted participants were excluded. Twenty participants were included in the study; $10(50 \%)$ were Black, 11 (55\%) were female, and the median time since last non-prescribed opioid use was 12 (interquartile range [IQR] 8-15) years (Table 1). The median length of interviews was $56 \mathrm{~min}$ (IQR, 48-60). Of note, the initial 15 interviews were conducted in person until March 2020; in response to the COVID-19 pandemic, the final 5 were conducted by telephone (May-July 2020).

\section{Disease Identity}

Two main themes of disease identity were described by participants or adopted during the interview: a living with addiction/chronic disease identity or a post-addiction/survivorship identity. These identities were the lens through which 
other aspects of their OUD explanatory model, including perspectives on the cause or etiology of OUD, role in maintaining recovery, and informed their perceptions of OAT (Fig. 1).

Living with OUD Identity. Some participants described an identity of living with OUD and as having a chronic disease. Many described themselves as an "addict" in the present tense. OUD was still present but now "quiet," "inactive," or "suppressed." Jeff (40s, White, male) used the symbolism of war where he was in a battle to suppress OUD when he said, "I always think of it like something cowering in the corner. Soon as you turn your back, it's going to come back... Right now I've got it controlled. It's trapped into the corner-away." Other participants maintained the living with OUD identity because it was accepted and reinforced in their social and mutual help networks. A common refrain was "once an addict, always an addict." For them, the experience of OUD is currently, and will always be, with them; therefore, they maintain the identity. Mike (40s, Black, male) explained it this way:

I was a user, so I always have that in the back of my mind what I did. It's like if you're a truck driver, just because you don't drive no more you still have that title.

Participants' disease identity appeared to inform their perceptions of the cause of their OUD. Participants who maintained a living with OUD identity more often described an internal cause for OUD. OUD (or an "addiction" in general) was something that they were born with or that was passed down through genes and family. Mary (40s, White, female) said:

My whole family — my father's brothers have had it - I know my uncle had a cocaine problem. I'm not the only one in the family who's had some sort of drug addiction problem, and that's why I sometimes truly believe it does run through the genes. If they could just remove that little addict part.
Similarly, the idea of an "addictive personality" was described by participants where a stable or immutable personality trait made them more likely to develop OUD. Aaron (40s, White, male) described it this way:

I just have an addictive personality. I am addicted to everything. Like there is a hole in me and I've got to fill it with something... Because I have gotten clean and then I started just buying DVDs. Not even joking, 8000 and counting.

When discussing the ongoing role of OAT, participants living with OUD identity more often described the continued use of OAT as necessary because they still have a disease requiring treatment. Many participants felt that the use of OAT was analogous to using medications for chronic medical conditions like diabetes. Other participants felt that the continued use of OAT and specifically the physiologic opioid dependence meant that they still had OUD. Cheryl (50s, Black, female) said:

I don't think I've gotten rid of it because I'm on methadone. And if my methadone was to be taken from me, I would get sick. And I would have no other choice but to go back to using.

For these participants, continuing OAT was acceptable despite the inconveniences of taking medication or needing to report to an OTP or clinic. Jason (40s, White, male) said:

I'm glad I'm taking it. Of course, with any medication, there are good things and bad things. On the one hand, yes, it helps with my opioid addiction, keeps it in check. But there are other problems... I guess, you have good and bad. I think that's not just with that medication (methadone). I mean, all of them have a good thing and a bad thing. So, but the big picture, I can maintain a somewhat normal life.

Finally, for participants with a living with OUD identity, their perspective of the OUD and need for ongoing

Table 1 Participant Characteristics

\begin{tabular}{|c|c|c|c|}
\hline Characteristics & All $(N=20)$ & Buprenorphine $(N=8)$ & Methadone $(N=12)$ \\
\hline Age, median years (IQR) & $46.5(42-52)$ & $43.5(38-49)$ & $48(44-52)$ \\
\hline Female, $n(\%)$ & $11(55 \%)$ & $5(63 \%)$ & $6(50 \%)$ \\
\hline \multicolumn{4}{|l|}{ Race, $n(\%)$} \\
\hline White & $10(50 \%)$ & $4(50 \%)$ & $6(50 \%)$ \\
\hline Black/African American & $10(50 \%)$ & $4(50 \%)$ & $6(50 \%)$ \\
\hline \multicolumn{4}{|l|}{ Clinic, $n(\%)$} \\
\hline OBOT & $7(35 \%)$ & $7(87 \%)$ & N/A \\
\hline OTP & $13(65 \%)$ & $1(13 \%)$ & $12(100 \%)$ \\
\hline Age at first non-prescribed opioid use, median years (IQR) & $21(17-24)$ & $20.5(18-25)$ & $21(17-23)$ \\
\hline Years since last non-prescribed opioid use, median years (IOR) & $12(8-15)$ & $10.5(8-13)$ & $13.5(8-18)$ \\
\hline Ever inject opioids, $n(\%)$ & $12(60 \%)$ & $4(50 \%)$ & $8(75 \%)$ \\
\hline
\end{tabular}




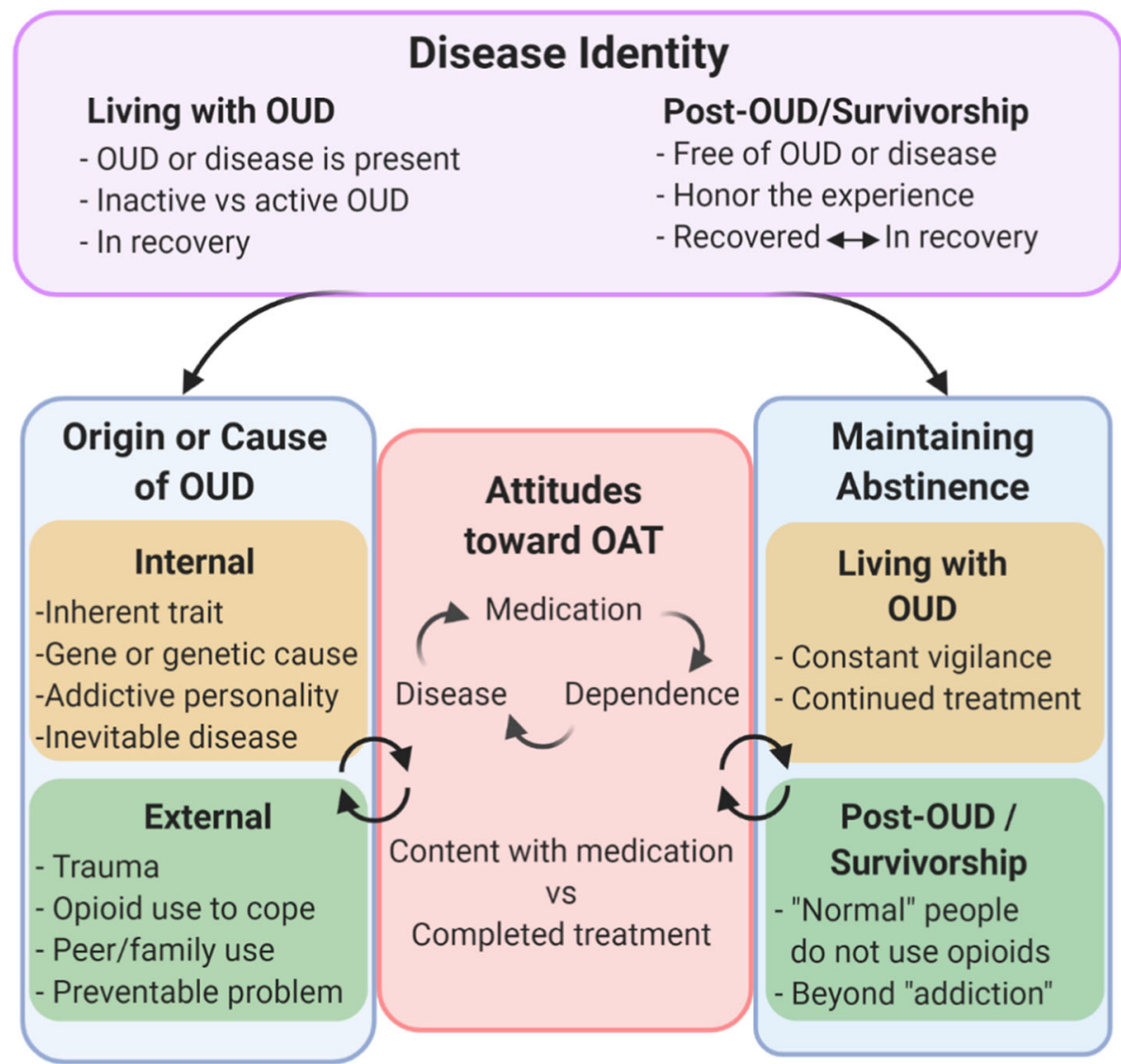

Figure 1 Conceptual framework relating opioid use disorder (OUD) disease identity, origin or cause of OUD, attitudes toward opioid agonist treatment (OAT), and maintaining abstinence.

treatment of a disease that is still present meant they maintained an awareness that they could easily return to previous behaviors and non-prescribed opioid use. Maintaining the living with OUD identity helped them remain vigilant and engage in behaviors to avoid temptation or thoughts of opioid use and maintain abstinence. Mike (40s, Black, male) described his vigilance the same way he always looks out when he is walking down the street: "I have a little saying, and I always say it, with one of my friends that's clean. I say that if I step off the curb the wrong way, I can be right back here."

Post-OUD/Survivorship Identity. Participants who described identities not related to OUD or previous drug use in general felt they had moved beyond their OUD. Some participants brought up the concept of being a "survivor" de novo while others who shared the "post-OUD state" identified with the survivorship identity when it was described.

Deborah (60s, Black, female) said:

It (OUD) is gone. It's released, believe me. I done burned it, threw it away. It's in the river. I don't know where it's at, but it isn't on me. And I would hope that other people that feel like that ball and chain is always going to be on their ankle the rest of their life. Once you free your mind from this disease, that ball and chain is gone.

Michele (30s, White, female) brought up the concept of survivorship de novo and compared her history of OUD with her mother's experience with breast cancer:

My mother fought breast cancer and she won. She always has a chance of getting it again so she lives in fear. I want to go out and conquer the day. I want to wake up today and just be normal so I can give my kids some kind of normal... I want to lead by example. I can say that I survived it (OUD).

Participants with a post-OUD/survivorship identity described a spectrum of perspectives of "being in recovery." Some describe a "void" or "hole" in their lives left by OUD that they needed to fill. These "voids" could be financial, personal relationships, or the feeling of lost time which required ongoing recovery activities. Katie (30s, White, female) said, "Recovery is not like one thing. It's not like the word 'Healed.' Recovery is not a one-day thing. Recovery is a lifetime thing." Others had moved past the active state of "recovery" and felt like they had "recovered." Tracy (30s, 
White, female) said:

I think my recovery is done. To me, recovery is like you just had surgery and this is your recovery time and then after that you're done. I think my recovery period was when I was in the extensive treatment program and then the inpatient and then the extensive outpatient; that was my recovery time. Now I've moved past it.

Many participants with a post-OUD/survivorship identity felt the chronic disease model of addiction kept them in a disease state resulting in continued stigmatization. Alice (60s, Black, female) had this to say about the "addict" label:

Once they removed all those things from their life, they no longer wanted that word associated with their life. Because addicts, that word, just 'addict' alone has so many bad stigmas to it... Once you use that word, people nose start to turn. And they don't even know what really an addict is... So I don't throw that word out there anywhere. It's my business and it's confidential to me... And it's not fair. I'm not that person that I used to be, and I worked hard to get to where I'm at. So how are you going to condemn me for something I did 10 years ago?

Participants who maintain a post-OUD/survivorship identity often described OUD as caused by something external to them. A common theme was trauma leading to opioid use and OUD. While the source and nature of the trauma varied, the use of non-prescribed opioids to cope was similar. Gloria (50s, Black, female) described a series of traumatic experiences including psychologic, physical, and sexual abuse perpetrated by a family member. In the aftermath of these events, she said, "It (opioid use) was taking my mind to a place where I didn't have to think about the bad stuff. It took me somewhere where everything felt good, and those things didn't bother me. It's a real peaceful feeling."

Similarly, participants reported using nonprescribed opioids to treat inadequately managed conditions like PTSD, depression, anxiety, or chronic pain. While these participants would describe the primary issue as internal to them-a disease, illness, or condition-the nonprescribed opioid use and subsequent OUD were rooted in their attempt to cope with symptoms. James (50s, Black, male) talked about the numerous injuries he sustained while doing construction work and said, "It (opioid use) kind of mask the problems that you have. So, if I were hurt my foot or my arm or something like that, I get a little heroin and I'm good to go." David (30s, white, male) described the role of opioid use to cope with the sadness of his father's death which marked the beginning of his struggle with depression:

I was down and sad of my dad passing. I was kind of like was like screw it. I'm like, you know, I don't care, you know, upset about that. So heroin is like, you know, it'll help me more than anything ... I guess it's cliché but like you know, it takes the pain away. By staying so high, I like, deal with it better.

Finally, participants described how opioids, specifically heroin, were ubiquitous in their community, peer groups, and families. They felt their OUD was a result of their environment. Some were introduced to heroin by family members. Others participated in the drug trade or used it socially with friends. Fred (30s, Black, male) summed it up when he described how, when he was growing up in Baltimore, heroin was the "in" thing, and had heroin not been so popular he would not have developed OUD. For these participants, heroin use in social settings led to physiologic dependence, subsequent withdrawal, and then continued use to avoid withdrawal symptoms, which was the point most identified as the start of their OUD.

Participants who identified with a post-OUD/survivorship state generally reported two attitudes toward ongoing OAT. First, some adopted a "if it's not broke, don't fix it" approach and were content with their treatment and did not feel compelled to make changes. Others questioned if OAT is currently needed or were actively tapering since they were past their OUD. Charles (60 years old, Black) said, "I'm trying to wean myself off that because I felt like it did what it did. It did everything it needed for me, and I don't need to have this ball and chain hooked to my leg anymore."

For participants with a post-OUD/survivorship identity, by not maintaining the addiction identity it removed the choice of using opioids and maintaining abstinence. Using nonprescribed opioids is something that they did in the past, but not now. Angela (50s, White, female) described it this way:

Because if you keep that thought that 'I'm an addict and I will always be an addict,' it's like it leaves a slit in a door where you can go back (to using opioids). Once you survived it, there is no going back. The door is closed now.

\section{Shifting and Evolving Perspectives}

Participants varied in how concrete or immutable their perspectives of OUD identity and explanatory model components were. Some participants who held starkly contrasting views of OUD identify (i.e., chronic disease vs post-OUD/survivorship) were consistent in their perspectives throughout the interview and across topics. For others, their perspectives changed as they contemplated their responses and were asked probing questions. A common occurrence is where participants would initially provide answers firmly based on the chronic disease model or information from mutual-help meetings and would then evolve through the interview. Some participants did not have the language to initially describe their current perspective on disease identity, but when offered 
the concept of survivorship, it would resonate with them and they would adopt that perspective. When Tina (50s, Black, female) heard the survivorship concept, she said "Exactly! I hadn't ever thought of it that way but that is exactly right." Still for others, their conflicting responses or contradictions lasted throughout the interview. When asked about this, a common refrain would be that "It depends." These participants described how their perspectives could be influenced by the current situation or people present and evolved to meet the needs of the moment.

\section{DISCUSSION}

In this qualitative study among patients in long-term, sustained remission from OUD, we explored perceptions of disease identity and how it is related to their explanatory model of OUD. The disease identity that participants held was closely tied to their beliefs about what caused their OUD. Participants who identified living with OUD/chronic disease model perceived the cause of their OUD was internal to them and required ongoing treatment and consistent vigilance. This perception is supported through research demonstrating the heritability of substance use disorders and the chronic disease model of OUD. ${ }^{22,23}$ Other participants identified with the post-OUD/survivorship identity which recognized that the experience of OUD is still with them but did not compel them to identify as living with OUD. This perspective is parallel to the cancer survivorship model which starts at the time of diagnosis and lasts the rest of a person's life and accounts for the general medical care needed after active cancer treatment is completed. ${ }^{21,24-26}$ Notably, participant responses were inconsistent and shifting at times which is consistent with Kleinman's observation that patient understanding of these representations "firms up in one situation only to dissolve in another."17

Primary care is increasingly where patients receive OUD and other substance use disorder treatment. ${ }^{27}$ This includes patients receiving OAT at an OTP that provides general medical and preventative care. ${ }^{18}$ While many professional organizations and government agencies promote the chronic disease model of OUD in order to reduce stigma and improve treatment acceptance, ${ }^{28-30}$ we found some participants reported that labeling OUD as a chronic disease "like diabetes"7 meant they were still viewed as an "addict." This label undermined the effort and progress they made in overcoming their OUD. The dissonance between clinician's chronic disease and patient's alternative perspectives could hamper the goals of patient-centered care and might promote further selfstigma and potentially result in worse treatment outcomes. ${ }^{31-35}$ When providing longitudinal care for these patients, our findings suggest that uncovering a patients' disease identity may help support patient-centered decision-making about long-term health, OUD treatment, and reduced patient perceptions of stigma. This is relevant since patients with substance use disorders continue to experience stigma in the healthcare system. ${ }^{36}$ The perspectives of patients in long-term, sustained remission from OUD are needed to inform a model of how to deliver care and what treatment recommendations are made in primary care settings.

Related to the longitudinal care of patients with OUD is the continued provision of OAT. The risk of returning to non-prescribed opioid use and overdose death is known to be the highest after medically supervised withdrawal and cessation of OAT. ${ }^{37}$ The current guidelines support offering indefinite OAT treatment to patients as long as the benefits outweigh risks and a current trial is exploring the life changes among patients with OUD. ${ }^{38,39}$ Among patients in long-term, sustained remission, understanding their perspectives on the cause of the disease and current identity could serve as a starting point for collaborative decision-making. In the context of routine care, a clinician might begin a discussion by adapting Kleinman's questions to elicit their patient's explanatory model. For example, a clinician might ask, "What do you most fear about OUD and its treatment with medication?" to better tailor options for continuation or tapering OAT. This approach could help reduce the adverse effects currently associated with the cessation of OAT.

There are some limitations to our study. First, our study results may not be generalizable to patients from different races and ethnicities given our study population reflected that of Baltimore's population which is $63 \%$ Black and $28 \%$ non-Hispanic White with people of all other races/ ethnicities making up $9 \%{ }^{40}$ Second, there is the risk that participants may exhibit a type of social desirability bias where they may use biomedical terms because they think is expected of them but may still hold perspectives that differ. ${ }^{41}$ This social desirability bias might be more significant for three of the participants who receive care from the study author conducting the interviews.

Future studies should include patients from different races, ethnicities, and/or geographic regions to provide further understanding of their explanatory models. Equally important is to understand the perceptions among patients with OUD in sustained remission who are not receiving OAT or engaged in traditional treatment or recovery activities. Finally, describing the perceptions of family members, friends, and clinicians who care for people in sustained remission could provide a comprehensive understanding of the long-term effects of OUD and improve the care of patients and their families.

\section{CONCLUSION}

We found that participants in long-term remission from OUD currently receiving OAT describing diverging and at times 
shifting descriptions of their disease identity, perceptions of the cause or origin of OUD, and perspectives on the chronic disease model of OUD. The results of this study suggest that a survivorship model of OUD may resonate with some patients and possibly reduce stigma in the healthcare setting. Furthermore, this study highlights the importance of understanding patients' explanatory model of their OUD to optimize patientcentered care.

Supplementary Information The online version contains supplementary material available at https://doi.org/10.1007/s11606-02106925-z.

Acknowledgements: We would like to acknowledge Research in Addiction Medicine Scholars (RAMS) faculty Jennifer Edelman, Michael Stein, Patrick O'Connor, Jeffery Samet, RAMS scholars, and Steven Harvey for their feedback on this research project. Figure created with BioRender.com.

Corresponding Author: Jarratt D. Pytell, MD, MHS; Department of Medicine, Division of General Internal Medicine, Johns Hopkins University School of Medicine, Baltimore, MD, USA

Funding JP was supported by the Research in Addiction Medicine Scholars Program (R25DA033211) and the National Heart, Lung, and Blood Institute (T32 HP10025). GC was supported by the National Institute on Alcohol Abuse and Alcoholism (K24 AA027483).

\section{Declarations:}

Conflict of Interest: The authors declare that they do not have a conflict of interest.

\section{REFERENCES}

1. Wilson N. Drug and Opioid-Involved Overdose Deaths - United States, 2017-2018. MMWR Morb Mortal Wkly Rep. 2020;69. doi:https://doi. org/10.15585/mmwr.mm6911a4

2. Substance Abuse and Mental Health Services Administration. Key Substance Use and Mental Health Indicators in the United States: Results from the 2018 National Survey on Drug Use and Health. Center for Behavioral Health Statistics and Quality, Substance Abuse and Mental Health Services Administration; 2019. Accessed July 30, 2020. https://www.samhsa.gov/data/

3. Schuckit MA. Treatment of Opioid-Use Disorders. N Engl J Med 2016;375(4):357-368. doi:https://doi.org/10.1056/NEJMra1604339

4. American Psychiatric Association. Diagnostic and Statistical Manual of Mental Disorders. Fifth Edition. American Psychiatric Association; 2013. doi:https://doi.org/10.1176/appi.books.9780890425596

5. Martins SS, Sarvet A, Santaella-Tenorio J, Saha T, Grant BF, Hasin DS. Changes in US Lifetime Heroin Use and Heroin Use Disorder: Prevalence From the 2001-2002 to 2012-2013 National Epidemiologic Survey on Alcohol and Related Conditions. JAMA Psychiatr 2017;74(5):445-455. doi:https://doi.org/10.1001/jamapsychiatry.2017. 0113

6. Boscarino JA, Hoffman SN, Han JJ. Opioid-use disorder among patients on long-term opioid therapy: impact of final DSM-5 diagnostic criteria on prevalence and correlates. Subst Abus Rehabil 2015;6:83-91. doi:https://doi.org/10.2147/SAR.S85667

7. U.S. Department of Health and Human Services (HHS), Office of the Surgeon General. Facing Addiction in America: The Surgeon General's Spotlight on Opioids. HHS; 2018. https://addiction.surgeongeneral.gov/ sites/default/files/Spotlight-on-Opioids_09192018.pdf. Accessed 11/ $02 / 2020$

8. Fiscella $\mathbf{K}$, Meldrum $\mathbf{S}$, Franks $\mathbf{P}$, et al. Patient trust: is it related to patient-centered behavior of primary care physicians? Med Care 2004;42(11):1049-1055. doi:https://doi.org/10.1097/00005650200411000-00003
9. Stewart M, Brown JB, Donner A, et al. The impact of patient-centered care on outcomes. J Fam Pract 2000;49(9):796-804.

10. Marchand $\mathbf{K}$, Beaumont $\mathbf{S}$, Westfall $\mathbf{J}$, et al. Conceptualizing patientcentered care for substance use disorder treatment: findings from a systematic scoping review. Subst Abuse Treat Prev Pol 2019;14(1):37. doi:https://doi.org/10.1186/s13011-019-0227-0

11. Meier PS, Barrowclough C, Donmall MC. The role of the therapeutic alliance in the treatment of substance misuse: a critical review of the literature. Addiction. 2005;100(3):304-316. doi:https://doi.org/10.1111/ j. 1360-0443.2004.00935.x

12. Fox AD, Masyukova M, Cunningham Co. Optimizing psychosocial support during office-based buprenorphine treatment in primary care: Patients' experiences and preferences. Subst Abus 2016;37(1):70-75. doi:https://doi.org/10.1080/08897077.2015.1088496

13. Drainoni M-L, Farrell C, Sorensen-Alawad A, Palmisano JN, Chaisson C, Walley AY. Patient Perspectives of an Integrated Program of Medical Care and Substance Use Treatment. AIDS Patient Care STDs 2014;28(2):71-81. doi:https://doi.org/10.1089/apc.2013.0179

14. Bassuk EL, Latta RE, Sember R, Raja S, Richard M. Universal Design for Underserved Populations: Person-Centered, Recovery-Oriented and Trauma Informed. J Health Care Poor Underserved 2017;28(3):896-914. doi:https://doi.org/10.1353/hpu.2017.0087

15. Institute of Medicine (US) Committee on Quality of Health Care in America. Crossing the Quality Chasm: A New Health System for the 21 st Century. National Academies Press (US); 2001. Accessed November 9, 2020. http://www.ncbi.nlm.nih.gov/books/NBK222274/.

16. Hampson SE, Glasgow RE, Toobert DJ. Personal models of diabetes and their relations to self-care activities. Health Psychol 1990;9(5):632-646. doi:https://doi.org/10.1037/0278-6133.9.5.632

17. De Maeyer J, Vanderplasschen W, Broekaert E. Quality of life among opiate-dependent individuals: A review of the literature. Int J Drug Policy 2010;21(5):364-380. doi:https://doi.org/10.1016/j.drugpo.2010.01.010

18. Leshner AI. Addiction is a brain disease, and it matters. Science. 1997;278(5335):45-47. doi:https://doi.org/10.1126/science.278.5335. 45

19. Volkow ND. What do we know about drug addiction? Am J Psychiatry 2005;162(8):1401-1402. doi:https://doi.org/10.1176/appi.ajp.162.8. 1401

20. Volkow ND, Koob GF, McLellan AT. Neurobiologic advances from the brain disease model of addiction. N Engl J Med 2016;374(4):363-371. doi:https://doi.org/10.1056/NEJMra1511480

21. American Society of Addiction Medicine. Public policy statement: definition of addiction 2019. Published September 15, 2019. Accessed October 2, 2020. https://www.asam.org/Quality-Science/definition-of-addiction.

22. Kleinman A. Patients and Healers in the Context of Culture: An Exploration of the Borderland between Anthropology, Medicine, and Psychiatry. University of California Press; 1980.

23. Kleinman A, Eisenberg L, Good B. Culture, Illness, and Care. Ann Intern Med 1978;88(2):251-258. doi:https://doi.org/10.7326/00034819-88-2-251

24. Shapiro CL. Cancer Survivorship. N Engl J Med 2018;379(25):24382450. doi:https://doi.org/10.1056/NEJMra1712502

25. Demers CH, Bogdan R, Agrawal A. The Genetics, Neurogenetics and Pharmacogenetics of Addiction. Curr Behav Neurosci Rep 2014;1(1):3344. doi:https://doi.org/10.1007/s40473-013-0004-8

26. Volkow ND, Muenke M. The genetics of addiction. Hum Genet 2012;131(6):773-777. doi:https://doi.org/10.1007/s00439-012-1173-3

27. Sun Y, Shigaki CL, Armer JM. Return to work among breast cancer survivors: A literature review. Support Care Cancer 2017;25(3):709-718. doi:https://doi.org/10.1007/s00520-016-3446-1

28. Hahn EE, Hays RD, Kahn KL, Litwin MS, Ganz PA. Post-traumatic stress symptoms in cancer survivors: relationship to the impact of cancer scale and other associated risk factors. Psychooncology. 2015;24(6):643652. doi:https://doi.org/10.1002/pon.3623

29. Yi JC, Syrjala KL. Anxiety and Depression in Cancer Survivors. Med Clin 2017;101(6):1099-1113. doi:https://doi.org/10.1016/j.mcna.2017.06. 005

30. Jones CM, Campopiano M, Baldwin G, McCance-Katz E. National and State Treatment Need and Capacity for Opioid Agonist MedicationAssisted Treatment. Am J Public Health 2015;105(8):e55-63. doi:https://doi.org/10.2105/AJPH.2015.302664

31. Luoma JB, Twohig MP, Waltz $\mathbf{T}$, et al. An investigation of stigma in individuals receiving treatment for substance abuse. Addict Behav 2007;32(7):1331-1346. doi:https://doi.org/10.1016/j.addbeh.2006.09. 008 
32. Crapanzano KA, Hammarlund R, Ahmad B, Hunsinger N, Kullar R The association between perceived stigma and substance use disorder treatment outcomes: a review. Subst Abus Rehabil 2018;10:1-12. doi:https://doi.org/10.2147/SAR.S183252

33. Street Jr. RL, Haidet P. How well do doctors know their patients? Factors affecting physician understanding of patients' health beliefs. J Gen Intern Med 2011;26(1):21-27. doi:https://doi.org/10.1007/s11606010-1453-3

34. Horowitz CR, Rein SB, Leventhal H. A story of maladies, misconceptions and mishaps: effective management of heart failure. Soc Sci Med 2004;58(3):631-643. doi:https://doi.org/10.1016/S0277-9536(03) 00232-6

35. Schouten BC, Meeuwesen L. Cultural differences in medical communication: A review of the literature. Patient Educ Couns 2006;64(1):21-34. doi:https://doi.org/10.1016/j.pec.2005.11.014

36. van Boekel LC, Brouwers EPM, van Weeghel J, Garretsen HFL. Stigma among health professionals towards patients with substance use disorders and its consequences for healthcare delivery: systematic review. Drug Alcohol Depend 2013;131(1-2):23-35. doi:https://doi.org/10. 1016/j.drugalcdep.2013.02.018

37. Wines JD, Saitz R, Horton NJ, Lloyd-Travaglini C, Samet JH Overdose after detoxification: A prospective study. Drug Alcohol Depend
2007;89(2):161-169. doi:https://doi.org/10.1016/j.drugalcdep.2006.12. 019

38. Substance Abuse and Mental Health Services Administration. Medications for Opioid Use Disorder. Treatment Improvement Protocol (TIP) Series 63. Publication No. PEP20-02-01-006. Substance Abuse and Mental Health Services Administration (US); 2020.

39. Ling W, Nadipelli VR, Ronquest NA, et al. Remission from chronic opioid use-Studying environmental and socio-economic factors on recovery (RECOVER): Study design and participant characteristics. Contemp Clin Trials 2019;76:93-103. doi:https://doi.org/10.1016/j.cct. 2018.11.015

40. Sorrell TR. Mexican Traditional Medicine: Application of a Traditional and Complementary Medicine System to Improve Opioid Use Treatment in Latinos. J Holist Nurs. Published online May 2, 2020:0898010120911540. doi:https://doi.org/10.1177/ 0898010120911540

41. Spradley JP. The Ethnographic Interview. Holt, Rinehart and Winston; 1979.

Publisher's Note: Springer Nature remains neutral with regard to jurisdictional claims in published maps and institutional affiliations. 